\title{
Chapter 3. Principles of treatment
}

\author{
Hypertension Research (2014) 37, 279-285; doi:10.1038/hr.2014.6
}

\section{POINT 3A}

1. Antihypertensive treatment should be performed to prevent the occurrence of cardiovascular disease due to sustained high blood pressure/progression/recurrence-related mortality and a reduction in the quality of life (QOL). (Recommendation grade $A)(I)$

2. Antihypertensive treatment should be indicated for all hypertensive patients with a blood pressure of $140 / 90 \mathrm{~mm} \mathrm{Hg}$ or above. These patients are stratified into three groups: low-, moderate- and high-risk groups, based on the blood pressure level, risk factors other than blood pressure and presence or absence of hypertensive organ damage.

3. Antihypertensive treatment consists of lifestyle modifications (step 1) and antihypertensive drug therapy (step 2). The starting point of this therapy should be determined on the basis of the risk level of individual patients.

4. The risk increases from the level of high-normal blood pressure. Progression from high-normal blood pressure to hypertension should be prevented by modifying the lifestyle. (Recommendation grade B) (II)

5. The goal of antihypertensive treatment is to reduce blood pressure to $<140 / 90 \mathrm{~mm} \mathrm{Hg}$. However, among diabetics and chronic kidney disease (CKD) patients with proteinuria, treatment should be performed in those with a blood pressure of $130 / 80 \mathrm{~mm} \mathrm{Hg}$ or more. The target blood pressure is $<130 / 80 \mathrm{~mm} \mathrm{Hg}$. In late-phase elderly persons, the target blood pressure is $<150 / 90 \mathrm{~mm} \mathrm{Hg}$. If tolerance is present, a blood pressure of $<140 / 90 \mathrm{~mm} \mathrm{Hg}$ should be targeted. (Recommendation grade A) (I)

6. In principle, antihypertensive drug therapy should be started with a low dose of a long-acting drug once a day. If the dose must be increased, twice-daily administration may be considered. Appropriate combination therapy with antihypertensive drugs should be administered to prevent adverse effects and enhance antihypertensive effects. Combination therapy should be considered from the outset for grade II or more severe hypertension. (Recommendation grade B) (III)

7. Reducing the number of tablets and the frequency of dosing is useful for improving adherence. (Recommendation grade $A$ ) (I)

8. Home blood pressure measurement is useful not only for the diagnosis of white coat hypertension and masked hypertension but also for evaluating the antihypertensive effects or for improving patients' adherence/concordance. (Recommendation grade A) (I)
9. Sufficient communication, information and consideration for QOL/adverse effects aid in improving adherence, achieving better blood pressure control and preventing cardiovascular disease. (Recommendation grade B) (Consensus IVa)

10. For treatment, the attending physician must eventually determine a therapeutic strategy after establishing concordance involving the results of epidemiological and clinical studies, clinical background of the patient, pharmacological actions of antihypertensive drugs, health expenditure and cost-effectiveness. (Recommendation grade $\mathrm{C} 1)(\mathrm{VI})$

\section{OBJECTIVES OF TREATMENT}

The objectives of antihypertensive treatment are to prevent the occurrence/progression/recurrence of cardiovascular disease related to sustained high blood pressure, reduce mortality and help patients with hypertension lead their lives as normally as do healthy people.

According to a meta-analysis of previous clinical studies, a $10-\mathrm{mm} \mathrm{Hg}$ decrease in systolic blood pressure and a $5-\mathrm{mm} \mathrm{Hg}$ decrease in diastolic blood pressure reduce the risks of stroke and coronary artery disease by $\sim 40(33-48 \%)$ and $20 \%(17-27 \%)$, respectively. ${ }^{226}$ In patients with a higher blood pressure level or at a more advanced age, antihypertensive drug therapy more markedly reduces these risks. ${ }^{123,227}$ An analysis of the results of a study involving patients with systolic hypertension also showed that a 10 $\mathrm{mm} \mathrm{Hg}$ decrease in systolic blood pressure reduced the risk of stroke by $30 \%$ or more and that of coronary artery disease by $20 \%$ or more. ${ }^{123,227-229}$

In Japan, the profiles of cardiovascular diseases differ from that in Europe and the United States. In men, the incidence of stroke is 3-6 times higher than that of myocardial infarction. In women, it is $4-12$ times higher than that of myocardial infarction. ${ }^{7}$ Therefore, the preventative effects of antihypertensive drug therapy on cardiovascular events may be more potent than in international studies. ${ }^{16,17,230-235}$

The effects of hypertension treatment increase with the risk of cardiovascular disease. ${ }^{236,237}$ Therefore, clinical studies involving higher-risk patients more strongly suggest the usefulness of treatment. The results of large-scale, placebo-controlled, randomized comparative studies in foreign countries have shown that antihypertensive drug therapy has many beneficial effects in patients with hypertension, and that it reduces the incidence and mortality rate of cardiovascular disease. ${ }^{238-240}$

The results of an analysis regarding the preventative effects of antihypertensive drug therapy on cardiovascular disease indicated that there was no gender difference in a treatment-related decrease in the risk for cardiovascular disease. ${ }^{16,241-243}$ 


\section{PATIENTS TO BE TREATED}

Antihypertensive treatment should be indicated for hypertensive patients of all ages. The HYVET study ${ }^{244}$ involving patients aged over 80 years showed that antihypertensive drug therapy decreased stroke-related mortality, cardiovascular morbidity, such as heart failure, and total mortality.

In patients with masked hypertension, such as morning hypertension, hypertension in the presence of stress and nighttime hypertension, antihypertensive treatment should be performed (see Chapter 2, Measurement and clinical evaluation of blood pressure). Basically, drug therapy is not performed in those with white coat hypertension, and the possibility that the condition may progress to hypertension in the future should be explained. Physicians must instruct patients to measure home blood pressure and improve their lifestyle. Periodic follow-up should be performed (see Chapter 2, Measurement and clinical evaluation of blood pressure).

\section{RISK STRATIFICATION FOR PROGNOSIS ASSESSMENT AND MANAGEMENT PLANS}

1) Risk factors for cardiovascular disease/organ damage

Hypertension is a primary risk factor for cardiovascular disease. In particular, it is the most important risk factor for stroke. Not only hypertension but also risk factors other than hypertension and the degree of organ damage such as hypertension-associated brain/heart/ kidney diseases are closely involved in the prognosis of hypertensive patients (Table 3-1). ${ }^{241}$ Therefore, in hypertension treatment, it is important to evaluate the blood pressure level, risk factors for cardiovascular disease (Table 3-1) and the presence or absence of organ damage/cardiovascular disease (Table 3-1).

As risk factors for cardiovascular disease other than blood pressure level, smoking, diabetes mellitus, dyslipidemia, obesity (especially visceral fat-type obesity), CKD, advanced age, and a family history of juvenile cardiovascular disease are important. Diabetes mellitus is an independent, strong risk factor. It is particularly important in comparison with other risk factors, as presented in Table 3-1. CKD and albuminuria are risk factors for cardiovascular disease, and are also classified as indicators of hypertensive organ damage. . $2,245-254^{2}$

Obese (especially visceral fat-type obesity) patients with a highnormal or higher blood pressure level, a fasting blood glucose level of $110 \mathrm{mg} \mathrm{dl}^{-1}$ or more, or 2 or 3 of three factors comprising dyslipidemia are regarded as having metabolic syndrome (MetS). As indicators of hypertensive organ damage or cardiovascular disease, the presence or absence of brain/heart/kidney/vascular/eyeground lesions should be evaluated (Table 3-1).

\section{2) Risk stratification}

In the Guidelines, hypertensive patients are stratified into three groups-low-, moderate- and high-risk groups-on the basis of the hypertensive level, primary risk factors (diabetes mellitus and other risk factors) and presence or absence of hypertensive organ damage/ cardiovascular disease, as shown in Table 3-2. In the JSH2009 Guidelines, risk stratification was also expressed with respect to patients with a high-normal blood pressure of 130-139/85-89 mm Hg. However, in the Guidelines, the column expression of high-normal blood pressure was eliminated. As emphasized in other sections, the risk in patients with a high-normal blood pressure is markedly higher than in those with an optimal blood pressure. In particular, patients with diabetes mellitus, CKD, MetS meeting all four items, three or more risk factors, organ damage or cardiovascular disease are regarded as high-risk patients. However, the category of high-normal blood pressure consists of a condition in which the target level of blood pressure
Table 3-1 Prognostic factors to be used for risk stratification for planning of hypertension management

Risk factors for cardiovascular disease other than blood pressure

Advanced age (65 years or older)

Smoking

\section{Dyslipidemia}

Hypo-HDL-cholesterolemia ( $<40 \mathrm{mg} \mathrm{dl}^{-1}$ )

Hyper-LDL-cholesterolemia ( $\geqslant 140 \mathrm{mg} \mathrm{dl}^{-1}$ )

Hypertriglyceridemia ( $\geqslant 150 \mathrm{mg} \mathrm{dl}^{-1}$ )

Obesity (BMI $\geqslant 25$ ) (especially visceral fat-type obesity)

Metabolic syndrome

Family history of juvenile cardiovascular disease (49 years or younger)

\section{Diabetes mellitus}

Fasting blood glucose $\geqslant 126 \mathrm{mg} \mathrm{dl}^{-1}$

2 -h post-loading blood glucose $\geqslant 200 \mathrm{mg} \mathrm{dl}^{-1}$

Casual blood glucose $\geqslant 200 \mathrm{mg} \mathrm{dl}^{-1}$

$\mathrm{HbA} 1 \mathrm{c} \geqslant 6.5 \%(\mathrm{NGSP})$

Organ damage/cardiovascular disease

Brain

Cerebral hemorrhage/cerebral infarction

Asymptomatic cerebrovascular disorders

Transient cerebral ischemic attacks

Heart

Left ventricular hypertrophy (electrocardiography and echocardiography)

Angina pectoris, myocardial infarction, after coronary reconstruction

Heart failure

Kidney

Proteinuria/albuminuria

Low eGFR ${ }^{\text {b }}\left(<60 \mathrm{ml} \mathrm{min}^{-1}\right.$ per $1.73 \mathrm{~m}^{2}$ )

CKD, established kidney disease (diabetic nephropathy and renal failure)

\section{Blood vessels}

Arteriosclerotic plaque

Carotid intima-media complex thickness $\geqslant 1.1 \mathrm{~mm}$

Macrovascular disease

Peripheral artery disease (low-ankle-brachial blood pressure ratio: $A B I \leqslant 0.9$ )

Eyeground

Hypertensive retinopathy

Abbreviations: CKD, chronic kidney disease; eGFR, estimated glomerular filtration rete; HDL, high-density lipoprotein; LDL, low-density lipoprotein.

aThe LDL cholesterol level is calculated using Friedwald's formula (TC - HDL-C - TG/5) by

fasting blood collection. When the TG level is $400 \mathrm{mg} \mathrm{dl}^{-1}$ or more or when blood is collected fasting blood collection. When the TG level is $400 \mathrm{mgdl}^{-1}$ or more or when blood is
after meals, non-HDL-C (TC-HDL-C) is used. Its criterion is LDL-C $+30 \mathrm{mg} \mathrm{dl}^{-1}$.

bThe eGFR is calculated using the following formula (eGFRcreat) with serum creatinine. When the muscular volume is extremely small, the following formula (eGFRcys) with serum cystatin is more appropriate.

eGFRcreat $\left(\mathrm{ml} \mathrm{min}^{-1}\right.$ per $\left.1.73 \mathrm{~m}^{2}\right)=194 \times \mathrm{Cr}^{-1.094} \times$ age $^{-0.287}($ women: $\times 0.739)$

eGFRcys $\left(\mathrm{ml} \mathrm{min}^{-1}\right.$ per $\left.1.73 \mathrm{~m}^{2}\right)=\left(104 \times\right.$ Cys $^{-1.019} \times 0.996$ age $($ women: $\left.\times 0.929)\right)-8$.

control is $<140 / 90 \mathrm{~mm} \mathrm{Hg}$ and that in which it is $<130 / 80 \mathrm{~mm} \mathrm{Hg}$. Descriptions based on the differentiation of these conditions may be complex, causing unnecessary confusion. In the Guidelines, a conventional target level of $130 / 85 \mathrm{~mm} \mathrm{Hg}$ for young and middle-aged persons was abandoned, and there is no group with this level. On the basis of these, the JSH2014-Preparing Committee decided to prepare figures and tables regarding risk stratification and planning of hypertension management at the initial examination only in patients diagnosed with hypertension to promote the clinical application of the Guidelines. Table 3-2 shows risk stratification in hypertensive patients. Figure 3-1 shows planning of hypertension management at 
Table 3-2 Stratification of the risk of cardiovascular disease based on clinic blood pressure

\begin{tabular}{|l|c|c|c|}
\hline $\begin{array}{l}\text { Classification of blood pressure } \\
\text { (prognostic factors } \\
\text { other than blood pressure) }\end{array}$ & $\begin{array}{c}\text { Grade I hypertension } \\
140-159 / 90-99 \mathrm{mmHg}\end{array}$ & $\begin{array}{c}\text { Grade II hypertension } \\
160-179 / 100-109 \mathrm{mmHg}\end{array}$ & $\begin{array}{c}\text { Grade III hypertension } \\
\geqslant 180 / \geqslant 110 \mathrm{mmHg}\end{array}$ \\
\hline $\begin{array}{l}\text { Risk I } \\
\text { (no prognostic factor) }\end{array}$ & Low risk & Moderate risk & High risk \\
\hline $\begin{array}{l}\text { Risk II } \\
\text { (either } 1 \text { to } 2 \text { risk factors other than diabetes mellitus } \\
\text { or MetS meeting } 3 \text { items is present) }\end{array}$ & Moderate risk & High risk & High risk \\
\hline $\begin{array}{l}\text { Risk III } \\
\text { (one of the following factors is present: diabetes mellitus, } \\
\text { CKD, organ damage/cardiovascular disease, MetS meeting } 4 \\
\text { items, or } 3 \text { or more risk factors) }\end{array}$ & High risk & High risk & High risk \\
\hline \begin{tabular}{l} 
Abbreviations: CKD, chronic kidney disease; MetS, metabolic syndrome, \\
\hline
\end{tabular}
\end{tabular}

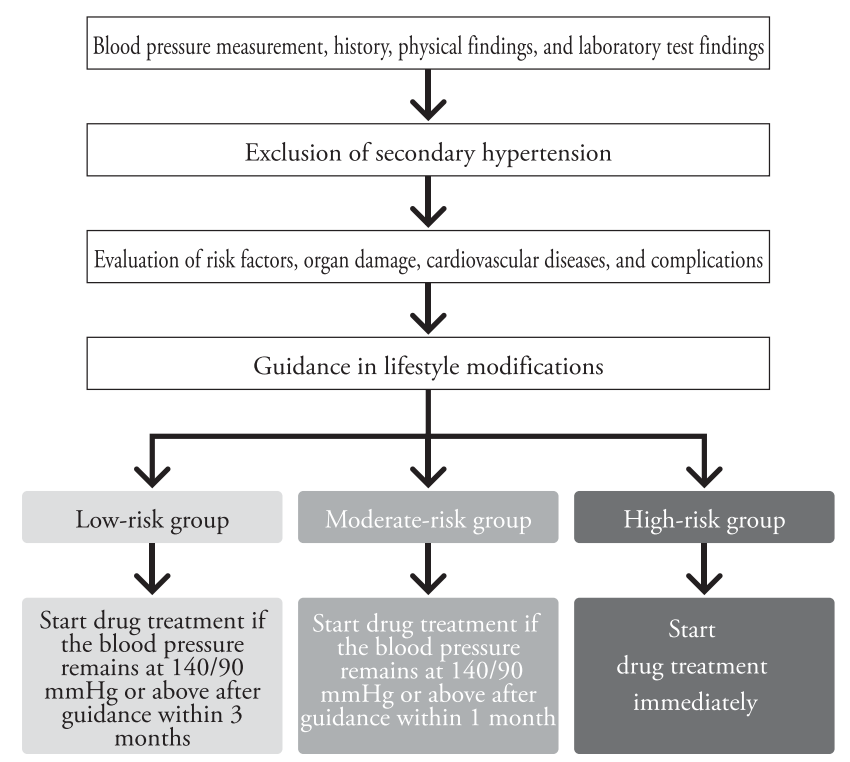

Figure 3-1 Planning of hypertension management at the initial examination. A full color version of this figure is available at the Hypertension Research journal online.

initial examination in these patients. Thus, hypertension-specified figures and tables may help physicians understand the Guidelines.

As the risk of cerebro-/cardiovascular diseases increases with the number of items meeting diagnostic criteria (see Chapter 7, Hypertension complicated by other diseases), MetS meeting three items (visceral fat-type obesity, high blood pressure and impaired glucose or lipid metabolism) was classified as Risk II, and MetS meeting all four items as Risk III.

When diabetes mellitus or CKD is present among risk factors, the risk is particularly high. ${ }^{252,253}$ On the basis of the results of many interventional studies, ${ }^{246,255-259}$ antihypertensive treatment is recommended in many guidelines. ${ }^{77,112,260}$

In the $2013 \mathrm{ESH} / \mathrm{ESC}$ Guidelines, ${ }^{120}$ the risk is stratified into four categories-low, moderate, high and super high-in accordance with risk factors, as described in the 2007 ESH-ESC Guidelines. ${ }^{260}$ However, therapeutic strategies are similar between high- and super-highrisk patients.
In the Japan Atherosclerosis Society Guidelines for Prevention of Atherosclerotic Cardiovascular Diseases 2012, a risk chart from NIPPON DATA is used with respect to the absolute risk of myocardial infarction-related mortality. ${ }^{261}$ The influence of hypertension on stroke is more marked than that on myocardial infarction among cardiovascular diseases. The Hisayama Study and Tanno-Sobetsu Study have also indicated that the JSH2009 risk stratification reflects the occurrence of cardiovascular disease in Japan; ${ }^{262,263}$ it may be clinically available. Considering the degree of recognition and availability in clinicians, the use of the conventional risk stratification table is recommended as the JSH2014-Preparing Committee's agreement.

In elderly persons in whom the absolute risk is high, the riskreducing effects of therapeutic intervention are relatively clear. ${ }^{123,227}$ However, in young and middle-aged hypertensive patients, the relative risk is higher, but the absolute risk is lower than in normotensive individuals of the same age. Therefore, the absolute risk-reducing effects of treatment do not appear. Long-term follow-up may be necessary.

\section{PLANNING OF HYPERTENSION MANAGEMENT AT THE INITIAL EXAMINATION}

If blood pressure is high at the initial examination, it is usually measured several times on another day to confirm a high blood pressure level. ${ }^{119,135,264-270}$ In addition, the patient is instructed to measure home blood pressure to confirm the presence or absence of white coat hypertension, white coat phenomenon and masked hypertension. The overall risk of the patient developing cardiovascular disease, including blood pressure, is assessed by investigating the presence or absence and degree of organ damage and risk factors for cardiovascular disease other than hypertension while eliminating secondary hypertension.

If home blood pressure or ABP differs widely from clinic blood pressure, it may be appropriate to determine a therapeutic strategy based on home blood pressure or ABP. ${ }^{119,135,264-270}$

After evaluating the overall risk of cardiovascular disease in hypertensive patients, the evaluation contents, therapeutic strategy and target control levels of clinic and home blood pressures should be explained to the patient until the patient understands them. In low-risk patients with a blood pressure of 140-159/90-99 $\mathrm{mm} \mathrm{Hg}$ (grade I hypertension) at the initial examination but no other risk factors, organ damage or cardiovascular disease, the lifestyle should be modified, and blood pressure should be measured again after a certain period (within 3 
months). Risk is stratified by blood pressure on additional measurement, and a therapeutic strategy is determined according to Figure 3-1. Therefore, even in low-risk patients, if blood pressure is not reduced to $<140 / 90 \mathrm{~mm} \mathrm{Hg}$ through lifestyle modifications alone, antihypertensive drug therapy is started after a certain period (within 3 months). On the other hand, even when blood pressure at the initial examination is classed as grade I hypertension, a therapeutic strategy matched to the risk should be established and executed according to Figure 3-1 if the risk is judged to be moderate or high on the basis of the number of risk factors for cardiovascular disease other than hypertension as well as on the basis of the presence or absence of organ damage, including MetS, diabetes mellitus, and CKD, or cardiovascular disease. If blood pressure at the initial examination is $160-179 / 100-109 \mathrm{~mm} \mathrm{Hg}$ (grade II), having excluded white coat hypertension or white coat phenomenon by measuring home blood pressure, and if the overall risk is judged to be moderate by risk assessment, antihypertensive drug therapy should be initiated after a period of lifestyle modification (within 1 month). Antihypertensive drug therapy should be initiated immediately if the risk is judged to be high even in patients showing grade II hypertension at the initial examination (Figure 3-1). If blood pressure is $\geqslant 180$ / $110 \mathrm{~mm} \mathrm{Hg}$ (grade III) at the initial examination, the risk is judged to be high and antihypertensive drug therapy must be initiated immediately (within a few days). These strategies should be applied in accordance with individual conditions. In particular, individualized strategies are required for elderly persons (see Chapter 8, Hypertension in the elderly).

In individuals with a high-normal blood pressure, progression to hypertension should be prevented by modifying their lifestyle. This reduces the risk for cardiovascular disease. ${ }^{271}$ In particular, patients with organ damage or other diseases such as diabetes mellitus, CKD, MetS meeting all items, cerebrovascular disorders and heart disease are judged to be at high risk even if their blood pressure is $<140$ / $90 \mathrm{~mm} \mathrm{Hg}$. In addition to guidance for lifestyle modifications (see Chapter 4, Lifestyle modifications), strict antihypertensive drug therapy should be promptly performed according to the target levels of blood pressure control matched to individual conditions (see Chapters 5, Treatment with antihypertensive drugs, Chapter 6, Hypertension associated with organ damage and Chapter 7, Hypertension complicated by other diseases).

Table 3-3 Target levels of blood pressure control

\begin{tabular}{lll}
\hline & Clinic blood pressure & Home blood pressure \\
\hline $\begin{array}{l}\text { Young, middle-aged and early- } \\
\text { phase elderly patients }\end{array}$ & $<140 / 90 \mathrm{~mm} \mathrm{Hg}$ & $<135 / 85 \mathrm{~mm} \mathrm{Hg}$ \\
Late-phase elderly patients & $<150 / 90 \mathrm{~mm} \mathrm{Hg}$ & $<145 / 85 \mathrm{~mm} \mathrm{Hg}$ \\
& $(<140 / 90 \mathrm{~mm} \mathrm{Hg}$ & $(<135 / 85 \mathrm{~mm} \mathrm{Hg}$ \\
& if toleranted) & if toleranted) \\
Diabetic patients & $<130 / 80 \mathrm{~mm} \mathrm{Hg}$ & $<125 / 75 \mathrm{~mm} \mathrm{Hg}$ \\
Patients with CKD & $<130 / 80 \mathrm{~mm} \mathrm{Hg}$ & $<125 / 75 \mathrm{~mm} \mathrm{Hg}$ \\
(with proteinuria) & & (criterion) \\
Patients with cerebrovascular & $<140 / 90 \mathrm{~mm} \mathrm{Hg}$ & $<135 / 85 \mathrm{~mm} \mathrm{Hg}$ \\
diseases & & (criterion) \\
Patients with coronary artery & & \\
disease & &
\end{tabular}

Abbreviation: CKD, chronic kidney disease.

Note: As diagnostic criteria for hypertension include a clinic blood pressure of 140/90 mm Hg and a home blood pressure of $135 / 85 \mathrm{~mm} \mathrm{Hg}$, the difference between the two values was applied to the difference between the target levels of clinic and home blood pressures.

\section{TARGET LEVELS OF BLOOD PRESSURE CONTROL}

On the basis of the results of interventional studies such as the MRC, ${ }^{272}$ HOT $^{273}$ and FEVER, ${ }^{274}$ the target level of blood pressure control should be established as $<140 / 90 \mathrm{mmHg}$ (Table 3-3). In young/middle-aged, low-risk patients with hypertension, the target level of blood pressure control had been established as $<130 /$ $85 \mathrm{~mm} \mathrm{Hg}$ in the JSH2009 Guidelines or earlier. This was similar to that described in the WHO-ISH1999 Guidelines. ${ }^{118}$ This target level was set on the basis of the results of the HOT Study, ${ }^{273}$ in which the cardiovascular prognosis was examined by classifying the target level of diastolic blood pressure into 90, 85 and $80 \mathrm{~mm} \mathrm{Hg}$ or less (three groups): there was no increase in cardiovascular risk in the lowest target level group, although there were no significant differences among the three groups. In addition, as the diastolic blood pressure level at which the incidence of cardiovascular disease reached a minimum in the subjects of the HOT Study overall was $82.6 \mathrm{~mm} \mathrm{Hg}$, the target level of blood pressure control has been established as optimal to normal blood pressure. As a result, until the JSH2009 Guidelines, there had been a gap between criteria for the start of antihypertensive drug therapy and the target level of blood pressure control. In the JSH2014 Guidelines, recommendations were decided on the basis of the results of comparative studies. As a result, a target level of $<140 / 90 \mathrm{~mm} \mathrm{Hg}$ may achieve a further blood pressure fall-related decrease in the relative risk for cardiovascular disease in young and middle-aged individuals. ${ }^{17}$ However, there are few interventional studies supporting a target blood pressure of $<140$ / $90 \mathrm{~mm} \mathrm{Hg}$ with significance; ${ }^{275}$ therefore, the target level of blood pressure control was established as $<140 / 90 \mathrm{~mm} \mathrm{Hg}$.

In diabetics and CKD patients with proteinuria in whom the risk for cardiovascular disease is high, the target level of blood pressure control is $<130 / 80 \mathrm{~mm} \mathrm{Hg}$ (see Chapter 6, Hypertension associated with organ damage and Chapter 7, Hypertension complicated by other diseases). In those with stroke or coronary artery disease, it is $<140 / 90 \mathrm{~mm} \mathrm{Hg}$ (see Chapter 6, Hypertension associated with organ damage). In late-phase elderly persons, in whom organ damage is frequently observed, the target level of blood pressure control should be established as $<150 / 90 \mathrm{~mm} \mathrm{Hg}$, and antihypertensive treatment must be carefully performed while monitoring changes in symptoms and laboratory data, considering the possibility of blood flow disturbance in important organs. Even in late-phase elderly persons, the final target level of blood pressure control is $<140 / 90 \mathrm{~mm} \mathrm{Hg}$.

Although evidence regarding home blood pressure-based interventional studies is not sufficient, the target levels of blood pressure control are $<135 / 85 \mathrm{~mm} \mathrm{Hg}$ in hypertensive patients and $<125 /$ $75 \mathrm{~mm} \mathrm{Hg}$ in hypertensive patients with diabetes mellitus according to the Ohasama Study 277,278 and observational studies with HOMED BP. ${ }^{279,280}$ Concerning other conditions, there is no evidence, but the target levels of home systolic and diastolic blood pressures are established as being 5-mm Hg lower than clinic systolic and diastolic blood pressures, considering differences in blood pressure on the home blood pressure-based diagnosis of hypertension.

With respect to the lower limit of the target level of blood pressure control, a further fall in blood pressure should be avoided in the presence of conditions ${ }^{281-286}$ in which excessive blood pressure fallrelated increases in the stroke-/myocardial infarction-/kidney hypofunction-related mortality rates were shown by a subanalysis of interventional studies involving high-risk hypertensive patients. Internationally, a meta-analysis ${ }^{287}$ of 61 prospective studies regarding the relationship between blood pressure and cardiovascular mortality indicated that cardiovascular mortality increased with an increase in blood pressure not only in hypertensive but also in normotensive 
individuals aged 40-89 years. However, it is unclear whether the incidence of cardiovascular disease further decreases at a blood pressure level of $<115 / 75 \mathrm{~mm} \mathrm{Hg}$. Treatment should be performed, considering that no interventional study has demonstrated an improvement in prognosis through a decrease in blood pressure to an optimal level.

\section{SELECTION OF TREATMENTS}

Several genetic and environmental factors are involved in the occurrence and progression of essential hypertension. Therefore, treatment always includes the correction of lifestyle (non-drug therapy), which comprises the greater portion of environmental factors. However, few patients achieve the target of blood pressure control through lifestyle modifications alone, and drug therapy is necessary in most cases. In individual patients, the risk should be stratified by comprehensively evaluating the blood pressure level, risk factors for cardiovascular disease and presence of cardiovascular disease, and a treatment plan must be established according to the stratification (Figure 3-1).

\section{1) Lifestyle modifications}

Hypertension is a lifestyle-related disease. Lifestyle modifications may prevent hypertension and exhibit blood pressure-decreasing effects. ${ }^{288-290}$ All patients with a high-normal or high blood pressure must undergo a change in lifestyle. In particular, when other lifestylerelated diseases such as dyslipidemia, diabetes mellitus, MetS and obesity are concomitantly present, lifestyle modifications are very important as a treatment method, and it is possible to simultaneously reduce these risk factors with safety at a low cost. To maintain the blood pressure-decreasing effects of lifestyle modifications, it is necessary for both physicians and patients to continue efforts over a long period. ${ }^{291}$

Although many hypertensive patients fail to achieve the target of blood pressure control through lifestyle modifications alone, it is possible to decrease the number and doses of antihypertensive drugs by enhancing their effects. Therefore, even after the start of antihypertensive drug therapy, the importance of lifestyle modifications does not change. ${ }^{292,293}$ The contents of lifestyle improvement are described in Chapter 4, Lifestyle modifications.

\section{2) Antihypertensive drug therapy}

Many patients with hypertension require drug therapy. Major antihypertensive drugs that are currently used include $\mathrm{Ca}$ antagonists, renin-angiotensin system inhibitors (angiotensin II receptor blockers (ARBs), angiotensin-converting enzyme (ACE) inhibitors and renin inhibitors), diuretics (thiazide and thiazide-like diuretics, loop diuretics and K-sparing diuretics) and $\beta$-blockers. In accordance with the condition, $\alpha$-blockers and central sympathetic nerve inhibitors (methyldopa, clonidine and guanabenz) are additionally administered. The action mechanisms and adverse effects of respective antihypertensive drugs are characteristic. On the basis of evidence on prognosis improvement from large-scale clinical studies, diuretics, Ca antagonists, ACE inhibitors and ARBs are selected as first-choice drugs (Chapter 5, Treatment with antihypertensive drugs).

For the administration of antihypertensive drugs, (1) a drug to be administered once a day should be selected; (2) drug therapy should be started at a low dose (in particular, commence therapy with a thiazide diuretic using half of the tablet); (3) when a 20-/10-mm Hg or greater decrease in blood pressure is targeted, combination therapy should be considered in the initial phase; (4) to enhance the depressor effects of drugs without adverse effects, drugs should be combined appropriately; (5) if a drug shows only a weak depressor effect or is poorly tolerated, it must be switched to another drug with a different action mechanism; and (6) positive indication should be considered in accordance with concomitant diseases or conditions, and antihypertensive drugs should be selected, considering contraindications/careful administration and interactions with combined drugs other than antihypertensive drugs.

\section{OTHER POINTS REQUIRING ATTENTION}

\section{1) Long-term treatment}

The objective of long-term treatment is to prevent cardiovascular disease and target organ damage by maintaining a target blood pressure level over a long period and comprehensively managing risk factors other than blood pressure.

As hypertension does not cause any marked symptoms, and as treatment continues over a long period, some patients may stop visiting medical facilities. An antihypertensive drug-related decrease in blood pressure is misunderstood as the cure for hypertension, and treatment is discontinued in some cases. ${ }^{294}$ Attending physicians should perform patient-involved treatment by sufficiently explaining the condition of hypertension, treatment methods, expected effects of treatment and adverse effects of antihypertensive drugs that may occur, through close communication with patients. In addition, it is important to make efforts/devise measures so that patients may observe lifestyle modifications and continue hospital visits and taking drugs. A good physician (hospital)-patient relationship must be maintained, and much attention should be paid so that antihypertensive treatment may not affect the patient's daily living or social activities. The sufficiency of communication with the physician and the degree of patient satisfaction with the medical staff markedly affect the patient's QOL. ${ }^{295}$

\section{2) Attention to the QOL}

Hypertensive patients' QOL is objectively and comprehensively evaluated on the basis of generalized comfort, physical symptoms, sexual activity, working efficiency, emotional state, intellectual functions, satisfaction with their lives, and social activities. ${ }^{296}$ Although the influence of hypertension on the QOL is less marked than that of other serious diseases, the QOL is impaired by being conscious of hypertension. ${ }^{297,298}$ Problems with emotional responses, home life, social activities, sleep and heart and digestive functions appear with increases in blood pressure. ${ }^{299}$ Furthermore, the QOL reduces with age, and there are marked individual differences among elderly persons. $^{300}$

Although treatment for hypertension improves the QOL, ${ }^{301,302}$ the adverse effects of antihypertensive drugs reduce it. ${ }^{303}$ As treatment for hypertension continues over a long period, it is important to maintain a favorable QOL for the continuation of treatment.

\section{3) Concordance/adherence}

The term 'compliance' (meaning obedience/acceptance) has been used to express the patient taking a drug and continuing treatment according to the physician's instructions. However, this means simply obeying regulations/orders and does not reflect what hypertension treatment should be. The entity 'adherence' (meaning support/ attachment) or 'concordance' (meaning agreement/harmony) was introduced. ${ }^{304,305}$ Adherence refers to the patient understanding the disease and necessity of treatment and continuing treatment spontaneously and positively, which is more desirable. In addition, the term concordance involves continuing to determine a therapeutic strategy based on an agreement through a partnership between the 
Table 3-4 Methods for the medical staff and patient to establish a partnership and continue concordance-based medical practice

- Talking with the patient about the risk of hypertension and advantages of treatment

- Providing information on hypertension treatment verbally, in writing and using video materials

- Determining the patient's life-matched therapeutic strategy based on the patient's agreement and subjective selection

- Decreasing the frequency of dosing and number of tablets to be taken by simplifying the regimen (use of a mixed preparation, one-package dispensing)

- Promoting self-measurement/recording of home blood pressure with a feedback of the results of assessment

- Establishing a treatment-supporting system involving the medical staff (physician, nurse, pharmacist, dietitian, public health nurse and care worker), patient and his/her family

- Talking with the patient about medical expenses

- Talking with the patient about reasons why he/she forgets to take drugs, particularly paying attention to adverse effects/anxiety/problems and changing the drug if necessary

physician and the patient on the assumption that the patient has sufficient knowledge of the disease and treatment. If the physician continues treatment from force of habit without sufficiently understanding the risk of hypertension or advantages and disadvantages of antihypertensive treatment, it may prevent the establishment of concordance. A supporting system by the medical staff, as a team, should be established through favorable communication between the physician (hospital) and the patient. Table 3-4 shows the ways through which to approach such adherence-/concordance-based medical practice.

Adherence to antihypertensive treatment is related to favorable/ unfavorable blood pressure control and the development/prognosis of cardiovascular disease. ${ }^{306-310}$ To achieve favorable concordance, it is necessary for the physician and the patient to understand the condition of hypertension, the objective of treatment (prevention of target organ damage and cardiovascular disease), treatment methods (lifestyle modifications and drug therapy), expected effects and adverse effects of treatment, and health expenditure. ${ }^{311-314}$ The appearance of adverse effects related to antihypertensive drugs affects adherence to antihypertensive treatment. ${ }^{315}$ When the number of tablets to be taken and frequency of dosing are smaller, adherence improves. ${ }^{307,316}$ In this sense, the use of a mixed preparation decreases the number of tablets to be taken and reduces drug expenses, improving adherence/concordance. ${ }^{317}$ Generic drugs approved through drug elution and biological equivalence studies (changes in the blood concentration of a drug after administration) may also improve adherence/concordance as drug expenses can be reduced.

\section{4) Blood pressure management in hypertensive patients taking antithrombotic drugs}

\section{POINT 3B}

Blood pressure management in hypertensive patients taking antithrombotic drugs

1. As hypertension is a risk factor for intracranial hemorrhage during therapy with antithrombotic drugs (antiplatelet drugs and anticoagulants), strict blood pressure control should be performed in patients taking these drugs. (Recommendation grade $B$ ) (II)
Recently, antiplatelet drugs have been increasingly used for the secondary prevention of arteriosclerotic disorders (transient cerebral ischemic attacks, cerebral infarction, coronary artery disease, peripheral arteriosclerotic disease and carotid sclerosis), and oral anticoagulants for the prevention of cardiogenic cerebral embolism/deep venous thrombosis. Treatment with these antithrombotic drugs increases the incidence of hemorrhagic complications, especially intracranial hemorrhage. ${ }^{318,319}$ After treatment with a drug-eluting stent, combination therapy with two antiplatelet drugs such as aspirin and clopidogrel is administered for a long period. To treat atherosclerotic disorders with atrial fibrillation, an antiplatelet drug is often combined with an oral anticoagulant. Such combination therapy with antithrombotic drugs further increases the risk of hemorrhage. ${ }^{319-323}$

As hypertension is a risk factor for intracranial hemorrhage during treatment with antithrombotic drugs, strict blood pressure management is important. The PROGRESS subanalysis involving patients taking antithrombotic drugs showed that the mean blood pressure in the antihypertensive drug group was $8.9 / 4.0 \mathrm{~mm} \mathrm{Hg}$ lower than that in the placebo group, and that the incidence of intracranial hemorrhage decreased by $46 \%{ }^{324}$ In the BAT prospective observational study involving Japanese patients taking antiplatelet drugs/ Warfarin, there was a correlation between the blood pressure level during therapy and incidence of intracranial hemorrhage, and the cutoff value of blood pressure for predicting the onset of intracranial hemorrhage was $130 / 81 \mathrm{~mm} \mathrm{Hg} .{ }^{325}$ Although evidence on the target level of blood pressure control to prevent hemorrhagic complications during therapy with antithrombotic drugs is not sufficient, blood pressure control should be further promoted carefully, considering a target level of $130 / 80 \mathrm{~mm} \mathrm{Hg}$, if possible, aiming at $<130 / 80 \mathrm{~mm} \mathrm{Hg}$, while monitoring ischemic symptoms/findings of important organs such as the brain/heart/kidney, in order to prevent intracranial hemorrhage in hypertensive patients taking antithrombotic drugs (with respect to patients with cerebral infarction, see Section 1 of Chapter 6, CEREBROVASCULAR DISEASE).

\section{5) Cost-effectiveness of antihypertensive treatment}

\section{POINT 3C}

\section{The cost-effectiveness of home blood pressure-based antihy- pertensive therapy with ARBs, ACE inhibitors, Ca antagonists} or diuretics, which are routinely used in Japan, is encouraging.

As economic burdens are required for chronic disease treatment, short-/long-term personal/social economic burdens must be considered in addition to the effects of treatment in clinical practice. Costeffectiveness analyses based on the results of large-scale clinical studies in Japan and other countries, as well as those using a model for predicting the prognosis, such as the Markov model, are useful.

As a method of cost-effectiveness analysis, the acquisition of 1QALY (quality-adjusted life year: survival in complete health status for 1 year) is used as a parameter of efficacy. ${ }^{326}$ In cost-effectiveness analyses, the results are commonly expressed using the incremental cost-effectiveness ratio (ICER) - that is, an additional cost required for test therapy in comparison with control therapy to obtain specific effects. When the ICER per 1QALY is 50000 dollars in the United States, 20,000 to 30,000 pounds in England and 5,000,000 yen in Japan, the cost-effectiveness is regarded as favorable. ${ }^{327,328}$ Expenses include not only those for antihypertensive drugs but also those for consultations, examinations and treatment for hypertension-related 
cardiovascular complications and concomitant diseases such as newonset diabetes mellitus.

Additional home blood pressure measurement ${ }^{329}$ and $\mathrm{ABPM}^{330}$ make it possible to rule out white coat hypertension in comparison with hypertension diagnosis based on clinic blood pressure alone. Therefore, their cost-effectiveness is favorable. According to the LIFE results on antihypertensive drug therapy, treatment with losartan as a first-choice drug was more effective than that with atenolol, reducing the cost. This treatment method was dominant. ${ }^{331}$

According to the results of the ASCOT-BPLA Study, combination therapy with amlodipine and perindopril, as a first-choice regimen, was more cost-effective than combination therapy with atenolol and bendroflumethiazide. ${ }^{332}$ According to the ALLHAT results, treatment with amlodipine, as a first-choice drug, was more cost-effective than therapy with chlortalidone. ${ }^{333}$

In an analytical model on the prognosis of Japanese patients with essential hypertension using the Markov model, ${ }^{334}$ the costeffectiveness of four treatment methods, ARBs (Ca antagonists were combined in some patients), ARBs (diuretics were combined in some patients), $\mathrm{Ca}$ antagonists (ARBs were combined in some patients) and diuretics (ARBs were combined in some patients), was analyzed. Assuming that their antihypertensive effects were similar, there were no marked differences in the cost or life-year among the four treatment methods in the absence of diabetes mellitus at the start of treatment. However, when diabetes mellitus was present at the start of treatment, ARBs (Ca antagonists were combined in some patients) exhibited maximal effects at a minimal cost. ${ }^{335}$ In a cost-effectiveness analysis of four treatment methods, monotherapy with ARBs, monotherapy with Ca antagonists, combination therapy with ARBs and Ca antagonists, and no antihypertensive drug, ${ }^{336}$ combination therapy with ARBs and Ca antagonists showed the greatest QALY in men without diabetes mellitus, followed by monotherapy with ARBs, monotherapy with Ca antagonists and the absence of treatment, as their antihypertensive effects differed. On the other hand, the cost was the lowest in untreated patients, followed by those receiving monotherapy with $\mathrm{Ca}$ antagonists, combination therapy with ARBs and $\mathrm{Ca}$ antagonists, and monotherapy with ARBs. The ICER of combination therapy with ARBs and Ca antagonists to the absence of treatment was 200,000 yen/ QALY. It was concluded that the cost-effectiveness of this combination therapy was favorable. ${ }^{337}$ In men with diabetes mellitus, this combination therapy showed the greatest QALY at a minimal cost. It was the most cost-effective among the four treatment methods.

In a study comparing a fixed combination drugs therapy with respective drugs a difference in adherence was observed. The fixed combination drugs entailed a lower cost of treatment. ${ }^{338}$ Thus, home blood pressure-based antihypertensive therapy with ARBs, ACE inhibitors, $\mathrm{Ca}$ antagonists or diuretics, which are routinely used in Japan, is cost-effective.

\section{Citation Information}

We recommend that any citations to information in the Guidelines are presented in the following format:

The Japanese Society of Hypertension Guidelines for the Management of Hypertension (JSH2014). Hypertens Res 2014; 37: 253-392.

Please refer to the title page for the full list of authors. 